\title{
DIARRÉIA AGUDA: 0 CONHECIMENTO MATERNO SOBRE A DOENÇA REDUZ 0 NÚMERO DE HOSPITALIZAÇÕES NOS MENORES DE DOIS ANOS?
}

\author{
* Lygia Carmen de Moraes Vanderlei, Gisélia Alves Pontes da Silva \\ Trabalho realizado no Instituto Materno Infantil de Pernambuco (IMIP), Recife, PE.
}

RESUMO - OBJETIVos. Analisar o conhecimento materno sobre as causas, sinais de desidratação e manejo da diarréia aguda e a ocorrência de hospitalização, por complicações desta doença, em seus filhos menores de dois anos.

Métodos. Desenho de corte transversal aninhado em estudo de caso - controle. Casos: crianças internadas por complicações da diarréia aguda no Instituto Materno Infantil de Pernambuco. Controles: crianças com doenças ambulatoriais exceto diarréia aguda. Variáveis: internamento por diarréia aguda (dependente); condições socioeconômicas; estado nutricional das crianças; tempo de aleitamento materno; conhecimento materno sobre diarréia aguda e seu manejo. $\mathrm{Na}$ análise foi utilizado software Epi-info 6.0.

RESULTADOS. Houve associação estatística entre internamento por diarréia aguda e condições socioeconômicas precárias $(p<0,01)$; desnutrição $(p<0,01)$; menor tempo de aleitamento materno $(p<0,01)$; desconhecimento materno sobre como evitar a desidratação $(p<0,05)$ e utilidade dos sais de reidratação oral $(p=0,02)$. Não houve associação estatisticamente significante para conhecimento da causa, dos sinais de desidratação e do manejo da diarréia.

CONCLUSÖEs. Houve associação entre hospitalização por diarréia aguda e más condições de vida, ausência de aleitamento materno, desnutrição, desconhecimento materno sobre como evitar a desidratação e sobre a utilidade dos sais de reidratação oral, sugerindo que a falta de conhecimento das mães reflete a adversidade de sua condição de vida.

UnITERMOS: Diarréia infantil. Hospitalização. Educação materna. Educação em saúde.

\section{INTRODUÇÃO}

A morbidade por diarréia é um indicador utilizado na avaliação do nível de saúde de uma população e varia de acordo com as diferentes regiões, localidades e grupos populacionais !. Essas variações regionais estão intimamente relacionadas com a profunda desigualdade de vida de seus habitantes referentes à renda, serviços públicos essenciais e à assistência à saúde e à educação?

Apesar do declínio do número de hospitalizações por diarréia aguda em todo o mundo com o advento da terapia de reidratação oral (TRO), implantada desde 1980 através do Programa de Controle da Doença Diarréica da Organização Mundial da Saúde, ${ }^{3}$ e do conseqüente decréscimo da mortalidade pela doença nos menores de cinco anos - de 3 para 1,8 milhões/ano na última década do século passado ${ }^{4}$, a diarréia ainda permanece como importante causa de hospitalização entre a população infantil.

\footnotetext{
*Correspondência:

Rua dos Coelhos, 300

CEP: 50.070-550 - Recife - PE - Brasil nepi@imip.org.br
}

No Nordeste brasileiro e no interior de Pernambuco a diarréia ainda é a primeira causa de hospitalizações entre as crianças, porém os municípios da Região Metro politana do Recife ela cede a principal posição para as pneumonias 5 .

Dentro do modelo explicativo da hospitalização por complicação da diarréia aguda, que inclui os determinantes socioeconômico, demográficos e biológicos, já bastante estudados e definidos, os fatores culturais são um outro importante aspecto a ser pesquisado, sendo de fundamental importância que não se perca de vista a interação dos fatores sócio-cognitivos, ou seja, o conhecimento de valores, atitudes e crenças da população de estudo $^{6}$, aqui representados pelas mães das crianças pesquisadas.

Sob este ponto de vista, deve ser priorizada a identificação das lacunas na atuação do profissional de saúde no tocante à sua atuação como educador em saúde, e o redirecionamento do atendimento enfatizando a transmissão de conhecimento para as mães, responsáveis pelo cuidado infantil, o que contribuiria para a melhoria do manejo da doença ${ }^{7,8}$.

Neste contexto, a estratégia de Atenção Integrada às Doenças Prevalentes na Infância
(AIDIPI), elaborada pela Organização Mundial da Saúde e Organização Pan-Americana de Saúde, em 1999, promove abordagens orientadas para o profissional na perspectiva da valorização do conhecimento materno acerca das doenças mais freqüentes na infância e seu manejo, como é o caso da doença diarréica?.

A pertinência do presente estudo reside no fato de que, à luz dos conhecimentos atuais, a doença diarréica infantil constitui-se no meIhor modelo em que o conhecimento e a atitude das mães, em relação ao adequado manejo da doença de seu filho, influencia efetivamente na redução das suas complicações ${ }^{10}$.

O objetivo deste estudo foi analisar o conhecimento materno sobre as causas, os sinais de desidratação e o manejo da diarréia aguda e a ocorrência de hospitalização, por complicações desta doença, em seus filhos menores de dois anos.

\section{Métodos}

A pesquisa foi desenvolvida no Hospital Geral de Pediatria do Instituto Materno-Infantil de Pernambuco (IMIP), Recife, PE, Brasil. A população de estudo foi constituída de todas as crianças menores de dois anos, acometidas de 
diarréia aguda, atendidas no Serviço de Pronto Atendimento do IMIP, no período de maio a outubro de 1997.

Realizou-se um estudo comparativo entre casos e controles, no qual os casos $(n=185)$ foram constituídos de todas as crianças menores de dois anos, internadas com sinais de desidratação conseqüente ao episódio diarréico agudo, com até cinco dias de duração. Os controles $(n=185)$ constituíram-se de crianças do mesmo grupo etário, com doenças ambulatoriais, exceto diarréia nas 48 horas que antecederam a consulta, que tivessem apresentado episódio diarréico agudo nos últimos quinze dias com resolução domiciliar, e que nunca foram hospitalizadas por diarréia.

A diarréia foi definida pela percepção da mãe sobre a presença da doença, "por terem perfeito conhecimento acerca do padrão normal de defecação dos seus filhos" "।.

A variável dependente foi ocorrência ou não de internamento por diarréia aguda; as variáveis independentes foram representadas por três tipos: a) socioeconômicas: características de moradia - tipo de paredes (tijolo ou taipa/tábua), tipo de piso (cerâmica/cimento ou terra/tábua); condições ambientais - esgotamento sanitário (fossa séptica ou fossa seca / céu aberto); b) biológicas - estado nutricional das crianças (índice altura/idade da curva do National Center for Health Statistics/NCHS), utilizadas na caracterização da amostra e c) variáveis culturais: tempo de aleitamento materno exclusivo e/ou predominante; conhecimento materno sobre a causa da diarréia modelo causal biológico (infecção, água ou alimentos contaminados); modelo alimentar (comida pesada, gordurosa, mal cozida); modelo climático (quentura, calor); modelo do desenvolvimento (erupção dentária) e causas místicas (susto, olhado); conhecimento materno dos sinais de desi dratação: olhos encovados, fontanela anterior deprimida, mucosa oral ressequida, diminuição/ausência de diurese, perda de peso (conhece 2-3 sinais; I; inadequados; não conhece); conhecimento materno de como evitar a desidratação, segundo o Ministério da Saúdel', "Oferecer soro reidratante oral ou caseiro após as evacuações, aumentar a oferta de líquidos e não modificar a dieta" (adequado; inadequado; não conhece) e do manejo do episódio e dieta" considerado correto quando não se modifica a dieta e se aumenta ou introduz a oferta de líquidos

Tabela I - Distribuição de freqüência das crianças segundo variáveis socioeconômicas

\begin{tabular}{|c|c|c|c|c|c|c|}
\hline \multirow[t]{3}{*}{ Variáveis } & \multicolumn{6}{|c|}{ Hospitalização por diarréia } \\
\hline & \multicolumn{2}{|c|}{ Sim } & \multicolumn{2}{|c|}{ Não } & \multicolumn{2}{|c|}{ Estatística } \\
\hline & $n(185)$ & $\%$ & $n(185)$ & $\%$ & $* \chi^{2}$ & $p$ \\
\hline \multicolumn{7}{|l|}{$\begin{array}{l}\text { Características de moradia } \\
\text { Paredes }\end{array}$} \\
\hline Tijolo & 138 & $(74,6)$ & 168 & $(90,8)$ & 15,8 & $<0,01$ \\
\hline Taipa/tábua & 47 & $(25,4)$ & 17 & $(9,2)$ & & \\
\hline \multicolumn{7}{|l|}{ Piso } \\
\hline Cerâmica/cimento & 146 & $(78,9)$ & 177 & $(95,7)$ & 21,29 & $<0,01$ \\
\hline Terra/tábua & 39 & $(21,1)$ & 8 & $(4,3)$ & & \\
\hline \multicolumn{7}{|l|}{$\begin{array}{l}\text { Condições ambientais } \\
\text { †Esgotamento sanitário }\end{array}$} \\
\hline Fossa séptica & 86 & $(58,9)$ & 107 & $(84,3)$ & 19,86 & $<0,01$ \\
\hline $\begin{array}{l}\text { Fossa seca /céu aberto } \\
\text { Nota: *correcão de Yates }\end{array}$ & 60 & $(4||)$, & 20 & $(15,7)$ & & \\
\hline
\end{tabular}

† Exclui "esgotamento ligado à rede geral" (146 casos; 127 controles)

Tabela 2 - Estado nutricional das crianças pelo índice altura/idade da curva do NCHS

\begin{tabular}{|c|c|c|c|c|c|c|}
\hline \multirow[t]{3}{*}{ Variáveis } & \multicolumn{6}{|c|}{ Hospitalização por diarréia } \\
\hline & \multicolumn{2}{|c|}{ Sim } & \multicolumn{2}{|c|}{ Não } & \multicolumn{2}{|c|}{ Estatística } \\
\hline & $n(185)$ & $\%$ & $n(185)$ & $\%$ & $* \chi^{2}$ & p \\
\hline$>$ percentil 10 & 95 & $(51,4)$ & 140 & $(75,7)$ & 27,61 & $<0,01$ \\
\hline Entre percentil 10 e 3 & 27 & $(14,6)$ & 22 & $(\mid 1,9)$ & & \\
\hline$<$ percentil 3 & 63 & $(34,0)$ & 23 & $(12,4)$ & & \\
\hline
\end{tabular}

Nota: * $c^{2}$ para tendência

durante a diarréia (cor reto / incorreto); conhecimento materno da utilidade, modo de preparo e oferta dos sais de reidratação oral (SRO) do Ministério da Saúde (correto; incorreto; não conhece).

Elaborou-se um formulário com questões pré-codificadas e abertas, testado e reajustado no estudo piloto. As entrevistas foram realizadas apenas com as mães das crianças estudadas, após seu conhecimento e consentimento prévios. A pesquisa foi aprovada pela Comissão de Ética em Pesquisa do IMIP.

Após a revisão e codificação dos formulários, as informações foram digitadas com dupla entrada no banco de dados criado para este fim. Na análise dos dados utilizou-se o teste do qui-quadrado de Pearson (ou o teste exato de Fisher, quando indicado) para verificar as diferenças de proporções. Para as variáveis categóricas ordenadas, foi calculado o teste do quiquadrado de tendência, por ter uma maior capacidade em detectar possíveis diferenças entre as categorias e verificar a existência de tendência entre os grupos. Com relação à variável aleitamento materno (diferença de mediana), realizou-se o teste de Kruskal-Wallis. O nível de confiança estatística considerado para a aceitação da hipótese de associação foi o de $95 \%$. Utilizou-se o pacote estatístico Epi-Info 6.0 em toda esta etapa.

\section{Resultados}

$\mathrm{Na}$ Tabela I, observaram-se condições de moradia mais favoráveis entre os controles que entre os casos, o mesmo ocorrendo com relação às condições ambientais, pelo maior percentual de crianças no grupo controle cujas residências possuíam fossa séptica, sendo as diferenças estatisticamente significantes.

A Tabela 2 evidencia que existiu um número de crianças desnutridas quase três vezes maior entre o grupo de casos que entre 0 de controles, com percentuais de $34 \%$ e 12\% respectivamente. A análise de tendência mostrou progressão do risco de hospitalização com o agravamento da situação nutricional $(p<0,01)$.

Para a variável tempo de aleitamento materno, a mediana de aleitamento exclusivo/ predominante entre os dois grupos de crianças 
Tabela 3 - Causas da diarréia segundo a opinião da mãe

\begin{tabular}{|c|c|c|c|c|c|c|}
\hline \multirow[t]{3}{*}{ Variáveis } & \multicolumn{6}{|c|}{ Hospitalização por diarréia } \\
\hline & \multicolumn{2}{|c|}{ Sim } & \multicolumn{2}{|c|}{ Não } & \multicolumn{2}{|c|}{ Estatística } \\
\hline & $\overline{n(185)}$ & $\overline{\%}$ & $\overline{n(185)}$ & $\overline{\%}$ & $\chi^{2}$ & $P$ \\
\hline Comida & 72 & 38,9 & 49 & 26,5 & 6,50 & 0,01 \\
\hline Dentes & 12 & 6,5 & 55 & 29,7 & 33,70 & 0,01 \\
\hline Gripe, virose, infecção & 7 & 3,8 & 9 & 4,9 & 0,26 & 0,61 \\
\hline Quentura & 8 & 4,3 & 7 & 3,8 & 0,07 & 0,79 \\
\hline Susto, olhado & 8 & 4,3 & 3 & 1,6 & 2,34 & 0,13 \\
\hline Medicamentos & 6 & 3,2 & 4 & 2,2 & 0,41 & 0,52 \\
\hline Vermes & 4 & 2,2 & 2 & 1,1 & *Fisher & 0,68 \\
\hline Água contaminada & । & 0,5 & 4 & 2,2 & *Fisher & 0,37 \\
\hline Contaminação de alimentos por moscas, sujeira & 2 & $\mathrm{I}, \mathrm{I}$ & I & 0,5 & *Fisher & 1,00 \\
\hline Outros & 8 & 4,3 & 7 & 3,8 & 0,07 & 0,79 \\
\hline Não sabe & 57 & 30,8 & 44 & 23,8 & 2,30 & 0.13 \\
\hline
\end{tabular}

Nota* Teste exato de Fisher $2 \mathrm{t}$

Tabela 4 - Conhecimento materno em relação aos sinais de desidratação

\begin{tabular}{|c|c|c|c|c|c|c|}
\hline \multirow[t]{3}{*}{ Variáveis } & \multicolumn{6}{|c|}{ Hospitalização por diarréia } \\
\hline & \multicolumn{2}{|c|}{ Sim } & \multicolumn{2}{|c|}{ Não } & \multicolumn{2}{|c|}{ Estatística } \\
\hline & $n(185)$ & $\%$ & $n(185)$ & $\%$ & $\chi^{2}$ & $P$ \\
\hline $\begin{array}{l}\text { Sinais de desidratação } \\
\text { De } 2-3 \\
\text { Apenas I } \\
\text { Inadequados } \\
\text { Não conhece }\end{array}$ & $\begin{array}{l}25 \\
99 \\
22 \\
39\end{array}$ & $\begin{array}{l}(13,5) \\
(53,5) \\
(11,9) \\
(21,1)\end{array}$ & $\begin{array}{l}22 \\
96 \\
47 \\
20\end{array}$ & $\begin{array}{l}(11,9) \\
(51,9) \\
(25,4) \\
(10,8)\end{array}$ & 0,33 & 0,56 \\
\hline
\end{tabular}

Nota: * $\chi^{2}$ para tendência

Tabela 5 - Conhecimento materno em relação a como evitar a desidratação, 0 manejo correto do episódio e da dieta

\begin{tabular}{|c|c|c|c|c|c|c|}
\hline \multirow[t]{3}{*}{ Variáveis } & \multicolumn{6}{|c|}{ Hospitalização por diarréia } \\
\hline & \multicolumn{2}{|c|}{ Sim } & \multicolumn{2}{|c|}{ Não } & \multicolumn{2}{|c|}{ Estatística } \\
\hline & $n(185)$ & $\%$ & $\overline{n(185)}$ & $\%$ & ${ }^{*} \chi^{2}$ & $P$ \\
\hline $\begin{array}{l}\text { †Evitar Desidratação } \\
\text { Adequado } \\
\text { Inadequado } \\
\text { Não conhece } \\
\text { +Manejo do episódio }\end{array}$ & $\begin{array}{l}75 \\
65 \\
43\end{array}$ & $\begin{array}{l}(41,0) \\
(35,5) \\
(23,5)\end{array}$ & $\begin{array}{l}89 \\
66 \\
28\end{array}$ & $\begin{array}{l}(48,6) \\
(36,1) \\
(15,3)\end{array}$ & $* 3,97$ & $<0,05$ \\
\hline $\begin{array}{l}\text { Correto } \\
\text { Incorreto } \\
\text { + Manejo da dieta }\end{array}$ & $\begin{array}{l}124 \\
61\end{array}$ & $\begin{array}{l}(67,0) \\
(33,0)\end{array}$ & $\begin{array}{l}139 \\
46\end{array}$ & $\begin{array}{l}(75,1) \\
(24,9)\end{array}$ & 2,58 & $=0,11$ \\
\hline $\begin{array}{l}\text { Correto } \\
\text { Incorreto }\end{array}$ & $\begin{array}{l}100 \\
85\end{array}$ & $\begin{array}{l}(54,1) \\
(45,9)\end{array}$ & $\begin{array}{l}108 \\
77\end{array}$ & $\begin{array}{l}(58,4) \\
(41,6)\end{array}$ & 0,54 & $=0,46$ \\
\hline
\end{tabular}

Nota: * $\chi^{2}$ para tendência; $†$ Correção de Yates; $¥ 2$ casos e 2 controles não responderam

foi de 30 dias (7-60 dias) para os casos e de 80 dias (45- I 20 dias) para os controles, resultados estatisticamente significantes $(p<0,0 I)$.

$\mathrm{Na}$ Tabela 3, relativa ao conhecimento materno sobre as causas de diarréia, notase que a maioria das mães entre os casos acreditava que $\mathrm{o}$ agente causal era a comida
(38,9\% contra $26,5 \%$ para os controles), enquanto que entre as mães dos controles a maior proporção encontrada foi para os dentes (29,7\% contra $6,5 \%$ para os casos), sendo estas diferenças estatisticamente significantes. A maioria das mães desconheciam a causa da diarréia, resultado seme-
Ihante entre os dois grupos (30,8\% contra $23,8 \%$ ). Os demais percentuais foram inexpressivos, sem significância estatística.

A Tabela 4, que se refere ao conhecimento materno dos sinais de desidratação durante a diarréia, mostra que os resultados não apresentaram diferença, com exceção dos dois últimos níveis, porém não houve associação estatística entre as variáveis.

A Tabela 5 exibe o conhecimento materno de como evitar a desidratação e manejar corretamente o episódio e a dieta, e mostra que o maior percentual de mães nos dois grupos tinha conhecimentos adequados de como evitar a desidratação. A análise de tendência sugeriu progressão do risco em direção ao desconhecimento materno, com significância estatística. Não houve associação estatística para o manejo do episódio e da dieta.

A Tabela 6 refere-se ao conhecimento materno sobre os sais de reidratação oral do Ministério da Saúde, e mostra que, com relação ao conhecimento de sua utilidade, houve maior percentual de respostas incorretas nos dois grupos, no entanto com freqüência menor no grupo controle. As demais variáveis não mostraram associação estatisticamente significante com a variável resposta.

\section{Discussão/ Conclusões}

Os diferentes aspectos relacionados com a doença diarréica infantil, desde o seu modo de contágio ao seu desfecho, cura ou agravamento, são explicados dentro de um modelo multicausal. Uma das formas de se investigar os determinantes deste agravo é por meio de estudos analíticos utilizando um modelo de hierarquização dos fatores envolvidos ${ }^{13}$. Em uma publicação anterior, a partir de dados da mesma população deste artigo, foram analisados os fatores de risco socioeconômico e demográficos e biológicos de uma forma hierárquica, através de análise multivariada ${ }^{15}$. Esse estudo evidenciou que os determinantes exclusivamente biológicos sofreram a influência dos socioeconômico e demográficos ${ }^{14}$.

No presente estudo, optou-se por enfatizar dentro de um modelo de multicausalidade, através de um estudo descritivo, as questões referentes ao conhecimento das mães a respeito da doença, seu modo de contágio, os sinais de agravamento e seu manejo adequado, que contribuíram para a 
Tabela 6 - Conhecimento materno da utilidade, modo de preparo e de oferta do SRO

\begin{tabular}{|c|c|c|c|c|c|c|}
\hline \multirow[t]{3}{*}{ Variáveis } & \multicolumn{6}{|c|}{ Hospitalização por diarréia } \\
\hline & \multicolumn{2}{|c|}{ Sim } & \multicolumn{2}{|c|}{ Não } & \multicolumn{2}{|c|}{ Estatística } \\
\hline & $n(185)$ & $\%$ & $n(185)$ & $\%$ & $* \chi^{2}$ & P \\
\hline $\begin{array}{l}\text { Utilidade } \\
\text { Correto }\end{array}$ & 42 & $(22,7)$ & 66 & $(35,7)$ & 8,0 & $=0,02$ \\
\hline Incorreto & $|3|$ & $(70,8)$ & 106 & $(57,3)$ & 0,0 & $-0,0 L$ \\
\hline Não conhece & 12 & $(6,5)$ & 13 & $(7,0)$ & & \\
\hline \multicolumn{7}{|l|}{ Preparo } \\
\hline Correto & 118 & $(63,8)$ & 102 & $(55,1)$ & 4,48 & $=0,11$ \\
\hline Incorreto & 26 & $(14, \mid)$ & 24 & $(13,0)$ & & \\
\hline Não conhece & 41 & $(22,2)$ & 59 & $(31,9)$ & & \\
\hline \multicolumn{7}{|l|}{ Oferta } \\
\hline Correto & 67 & $(36,2)$ & 62 & $(33,5)$ & 0,33 & $=0,85$ \\
\hline Incorreto & 97 & $(52,4)$ & 100 & $(54,1)$ & & \\
\hline Não conhece & 21 & $(11,4)$ & 23 & $(12,4)$ & & \\
\hline
\end{tabular}

redução das complicações, dentre elas a desidratação e o internamento. É importante que seja considerado que o método mais adequado para a abordagem das variáveis culturais seria o qualitativo, mas o estudo em tela poderá fornecer pistas para pesquisas posteriores.

Com referência às variáveis socioeconômicas, a análise dos dados mostrou associação entre hospitalização por diarréia aguda (DA) e adversidade de condições de vida das crianças, sendo esta relação demonstrada em vários estudos $5,15,16,17,18$. As crianças hospitalizadas apresentaram um percentual de desnutrição crônica aproximadamente três vezes maior do que as do grupo de controles, dados que apontam para o sinergismo existente entre baixa idade, desnutrição e evolução desfavorável do episódio diarréico ${ }^{15,19}$.

$\mathrm{O}$ aleitamento materno, considerado no estudo como variável cultural, foi também analisado como fator de risco, pelo seu documentado efeito protetor no desfecho favorável do episódio diarréico ${ }^{15,16,17}$, estando os nossos achados de acordo com a literatura mundial, uma vez que a mediana de aleitamento entre as crianças do grupo controle foi superior à encontrada entre as do grupo de casos. Por outro lado, deve se valorizar o comportamento materno no que diz respeito ao cuidado infantil, e a decisão de amamentar reflete este envolvimento, pela repercussão positiva nas condições de saúde da criança nos primeiros anos de vida ${ }^{19}$.

Quanto à causa da diarréia, as opiniões das mães guardaram semelhança com estudos anteriores $^{20-23}$. Dentro dos diferentes modelos explicativos da doença diarréica (que caracterizam o significado que as pessoas atribuem à doença e como tentam explicá-la), estão incluídos o modelo biológico, o ali mentar, o climático, o do desenvolvimento e o de causas místicas ${ }^{21-24}$, dentre outras diferentes classificações.

Segundo o modelo biológico, que é o adotado pela clínica médica, a causa necessária para que ocorra a diarréia é o contágio com o enteropatógeno, cuja via de transmissão é, na maioria dos casos, fecal/oral ${ }^{20-22}$. O conhecimento materno concordante com esse modelo obteve um percentual semelhante e inexpressivo nos grupos, sugerindo o desconhecimento por parte da maioria das mães do mecanismo de contágio da doença diarréica.

O maior percentual de causa de diarréia atribuído à erupção dentária, correspondente ao modelo do desenvolvimento, encontrado entre os controles se deveu provavelmente à presença de episódios mais leves da doença neste grupo, que são considerados pelas mães como "normais", fazendo parte do crescimento e desenvolvimento das crianças, concomitante com o surgimento dos dentes ${ }^{24}$. Sodemann et al. relatam uma redução de $80 \%$ na intenção das mães procurarem serviço de saúde quando achavam que a diarréia estava associada à erupção dentária, provavelmente em decorrência de serem quadros mais leves ${ }^{25}$.

É importante o achado de que um percentual maior de mães entre os casos atribuiu à doença causas sobrenaturais, como "mau olhado; medo de fogos; susto", que corresponde ao modelo místico. Este resultado sugere uma maior ignorância sobre o modo de transmissão da doença por parte das mães das crianças hospitalizadas, uma vez que, diferente da erupção dentária que encontra algum paralelismo com a maior exposição das crianças ao contágio pela deambulação, a idéia do misticismo para a explicação dos fatos biológicos aponta para os estágios mais elementares do conhecimento humano, em geral com nenhum contato com a ciência médica formal ${ }^{25}$.

Faz-se necessário que os profissionais de saúde conheçam os diferentes modelos explicativos da doença diarréica, o que os capacitará no reconhecimento do seu significado na percepção das mães para que possam criar mecanismos que facilitem a comunicação através de mensagens educativas aceitas por elas no sentido da prevenção da doença e do seu adequado manejo $0^{26,27}$.

O conhecimento materno dos sinais de desidratação foi semelhante entre os grupos, sugerindo que não houve uma compreensão da evolução da gravidade do episódio por parte das mães. Porém, o percentual um pouco maior de respostas corretas encontrado para as mães das crianças internadas pode estar indicando uma maior facilidade delas em descrever os sinais no momento de sua ocorrência, por ocasião da entrevista. Por outro lado, as mães do grupo controle provavelmente nunca manejaram um episódio de desidratação, pois seus filhos não tiveram internamento anterior por doença diarréica aguda.

Leal et al. ${ }^{28}$ relatam que mães que já haviam vivenciado um episódio de desidratação anterior não foram capazes de, no episódio atual, reconhecer que seus filhos apresentavam desidratação e concluíram que isso "ocorre porque o repasse de informação no serviço de saúde foi nulo ou inadequado". Yoder e Hornik ${ }^{29}$, analisando dados de dez inquéritos epidemiológicos em diferentes regiões da África e da Ásia, chamam a atenção para o fato das mães basearem a decisão de procurar um serviço de saúde quando as crianças apresentam vômitos, febre e comprometimento do estado geral. A não valorização das perdas diarréicas e dos sinais precoces de desidratação contribuiria para a acentuação do quadro e conseqüentemente para o aumento do número de hospitalizações. 
Entretanto, se as mães do grupo controle desconheciam os sinais, os dados sugerem um melhor manejo por parte delas, pois houve um percentual maior de conhecimento materno sobre como evitar a desidratação e manejar corretamente o episódio e a dieta entre as mães desse grupo, apesar de apenas ter existido diferença estatística para a primeira variável.

Analisando-se os dados referentes ao uso do SRO, observou-se que a maioria das mães não tinha conhecimentos adequados sobre a sua utilidade em prevenir ou tratar a desidratação, aspecto evidenciado pelo grande percentual de respostas incorretas, com apenas $29 \%$ mostrando conhecimentos adequados, mesmo que esses tenham sido superiores para as mães do grupo controle. Os resultados mostram a necessidade de uma maior informação às mães, para que essas conheçam a eficácia do SRO na prevenção e no combate à desidratação, sendo a crença errônea de que o mesmo interrompe a diarréia, um grande obstáculo à sua utilização, pois leva a uma imagem negativa da TRO na opinião materna, pela sua frustração ao constatar a permanência da doença em seu filho na vigência do uso dos sais7,7,30.

Apesar do percentual maior de mães que sabiam preparar corretamente o SRO, a sua oferta foi realizada de maneira incorreta por mais da metade delas, independente do grupo. Nesta pesquisa, os resultados mostram que, mesmo com a melhoria dos conhecimentos maternos sobre a TRO ao longo de mais de uma década, é necessário um maior empenho por parte dos profissionais de saúde, no sentido de se criarem estratégias para que a informação seja transmitida às mães de maneira mais eficiente ${ }^{7,8,31}$.

O estudo demonstrou a existência de associação entre hospitalização por diarréia aguda em menores de dois anos e precariedade de condições de vida, ausência de aleitamento materno e desnutrição. A garantia que o grupo de controles tivesse a mesma probabilidade de ser exposto que o de casos foi atendida uma vez que os dois grupos eram formados por casos incidentes, dentro dos mesmos requisitos, com exceção que para os controles não poderia ter havido hospitalização prévia por diarréia, nem após o episódio em tela, não afastando uma ocorrência futura.

O desconhecimento sobre a doença e seu manejo foi encontrado em ambos os grupos estudados. A falta de conhecimento materno sobre o manejo correto do episódio diarréico sugere que esse desconhecimento reflete sua condição de pobreza, de baixo nível educacional, de pouco acesso aos serviços de saúde, de exclusão social, visto que apesar da população de estudo ter se constituído de casos incidentes, os menos favorecidos economicamente, desnutridos, com mediana de aleitamento menor, cujas mães desconheciam o manejo correto do episódio diarréico, tiveram como desfecho a hospitalização.

Nesta pesquisa, não se perdeu de vista a possibilidade da existência de variáveis de confundimento, além das que foram consideradas no desenho, que podem ter contribuído total ou parcialmente para o aparente efeito das exposições estudadas sobre o desfecho final observado, ou mesmo terem ocultado a verdadeira associação. Porém, os resultados apontam para a necessidade de serem levantadas novas abordagens de investigação da doença diarréica.

\section{Conflito de interesse: não há.}

\section{SUMMARY}

\section{ACUTE DIARRHEA: DOES MOTHER'S KNOWLEDGE OF THE DISEASE REDUCE ADMIS- SION OF CHILDREN UNDER TWO YEARS OF AGE?}

OBjeCTIVES. To analyze the relationship of mother's knowledge about signs of dehydration, causes and management of acute diarrhea with admission of children under two years of age with the disease.

METHODS. Anested cross-sectional and casecontrol study was carried out with children who were admitted at the "Instituto Materno Infantil de Pernambuco" with acute diarrhea: and control children admitted with other diseases. Variables were: patient admission having or not acute diarrhea (dependent); social-economic condition, nutritional state; duration of breastfeeding; mother's knowledge about acute diarrhea and its management (independent). The Epi-info software, 6.0 was used for analysis.

RESULTS. There was statistical association between patient admission with acute diarrhea and poor social-economic conditions $(p=0.01)$; malnutrition $(p=0.01)$; short time of breastfeeding $(p=0.01)$, lack of mother's knowledge about how to avoid dehydration $(p=0.05)$ and effectiveness of oral rehydration solutions $(p=0.02)$. There was no statistical association with knowledge about causes, signs of dehydration or management of acute diarrhea.

Conclusions. The study disclosed association between admission of children under two years of age with acute diarrhea and poor social-economic conditions; short time of breastfeeding, malnutrition, mother's lack of knowledge on how to avoid dehydration and effectiveness of oral rehydration solutions. Data suggests that lack of mother's knowledge reflects poor socialeconomic conditions. [Rev Assoc Med Bras 2004; 50(3): 276-8I]

KEY wORDs: Infantile diarrhea. Patient admission. Mother's knowledge. Health education.

\section{REFERÊNCIAS}

I. Alam N, Henry FJ, Rahaman MM. Reporting errors in one-week diarrhoea recall surveys: experience from a prospective study in rural Bangladesh. Int J Epidemiol | 989; | 8:697-700.

2. Benício MHA, Cesar CLG, Gouveia NC. Perfil de morbidade e padrão de utilização de serviços de saúde das crianças brasileiras menores de cinco anos - 1989. Brasília (DF): INAN/Instituto Nacional de Saúde e Nutrição; 1990.

3. WHO. World Health Organization. Programme for Control of Diarrhoeal Diseases: report 1984-1985. Geneve; 1986. (Fifth Programme, |6:28-31).

4. WHO. World Health Organization. The World health report. Geneve; 1999.

5. INAN/Instituto Nacional de Alimentação e Nutrição, IMIP/Instituto Materno Infantil de Pernambuco, UFPE/Universidade Federal de Pernambuco, Recife. Secretaria de Saúde. II Pesquisa Estadual de Saúde e Nutrição: saúde, nutrição, alimentação e condições socioeconômicas no Estado de Pernambuco: 1997. Recife; 1998.

6. Minayo MCS. Abordagem antropológica para avaliação de políticas sociais. Rev Saúde Pública 1991; 25:233-8.

7. Bentley ME. The household management of childhood diarrhea in rural north India. Soc Sci Med 1988; 27:75-85.

8. Hounsa AM, Godin G, Alihonou E, Valois P. Girard J. Na application of Ajzen's theory of planned behaviour to predict mother's intention to use oral rehydration therapy in a rural area de Benin. Soc Sci Med 1993; 37:253-61.

9. Ministério da Saúde, Organização Mundial da Saúde, Organização Pan-Americana da Saúde. Atenção integrada às doenças prevalentes na infância: guia do facilitador para os módulos: curso de capacitação módulo 8. Brasília (DF); 1999. 
10. López SL, Hernández FM. Manual de consulta educativa en diarreas. México: Hospital Infantil de México Federico Gómez; 1994.

II. Moore HA, De La Cruz L, Vargas OM. Diarrhoeal disease studies in Costa Rica. Morbidity and mortality from diarrhoea. Am J Epidemiol 1965; 82:|43-6|

12. Ministério da Saúde. Secretaria Nacional de Políticas de Saúde, Departamento Nacional de Saúde Materno Infantil. Programa de Assistência Integral à Saúde da Criança (PAISC). Manual de ações básicas na assistência integral à saúde da criança. Brasília (DF); 1987.

13. Kirkwood BR. Essentials of medical statistics. Oxford: Blackwell Science; 1988.

14. Vanderlei LCM, Alves GAP, Braga JU. Fatores de risco para internamento por diarréia aguda em menores de dois anos: estudo de casocontrole. Cad Saúde Pública 2003; 19: 455-63.

15. Fuchs SC, Victora CG, Martines J. Case control study of risk of deydrating diarrhoea in infants in vulnerable period after full weaning. Br Med J 1996; 313:342-7.

16. Fuchs SC, Victora CG. Risk and prognostic factors for diarrheal disease in Brazilian infants: a special case - control design application. Cad Saúde Pública 2002; 18:773-82.

17. Claeson JG, Merson MH. Global progress in the control of diarrhoeal diseases. Pediatr Infect Dis J 1990; 9: 345-55.
18. Rego RCF. Destino dos dejetos, lixo e diarréia infantil em uma comunidade periurbana de Salvador, Bahia, 1996 [dissertação]. Salvador: Instituto de Saúde Coletiva da Universidade Federal da Bahia; 1996.

19. Islam MA, Rahman MM, Mahalanabis D, Rhaman AK. Death in a diarrhoeal cohort of infants and young children soon after discharge from hospital: risk factors and causes by verbal autopsy. J Trop Pediatr 1996; 42: 342-7.

20. Victora CG, Smith PG, Vaughan JP, Nobre LC, Lombardi C, Teixeira AMB, Barros, FC. Infant feeding and deaths due diarrhoea - a case - control study. Am J Epidemiol 1989; | 29: | 032-4|.

21. Manz F, Hof MA, Haschke F. The motherinfant relationship: who controls breastfeeding frequency? Lancet 1999; 353: I | 52-6.

22. Zoysa I, Carson D, Feachem R. Perception of childhood diarrhoea and its treatment in rural Zimbabwe. Soc Sci Med 1984; 19:727-34.

23. Green EC. Traditional healers, mothers and childhood diarrhoeal disease in Swaziland: the interface of anthropology and health education. Soc Sci Med 1985; 20:227-85.

24. Weiss MG. Cultural models of diarrheal illness: conceptual framework and review. Soc Sci Med 1988; 27:5-16

25. Sodemann M, Jakobsen MS, Molbak K, Martins C, Aaby P. Maternal perception of cause, signs and severity of diarrhoea in a suburban west African community. Acta Paediatr 1996; 85: 1 062-4.

26. Feliciano KVO, Kovacks MH. Organização das práticas de saúde e vulnerabilidade à diarréia infantil. J Pediatr (Rio de J) 2000; 76:27-36.

27. Desjeux D, Favre I, Simongiovanni J. Why is oral therapy associated with drugs in the treatment of diarrhoea? J Pediatr Gastroenterol Nutr 1996; 22: I 12-9.

28. Leal MC, Silva RI, Gama CGN. Percepção materna da desidratação em crianças com diarréia. Estudo de concordância com o diagnóstico médico. Rev Saúde Pública 1990; 24:196-203.

29. Yoder PS, Hornik RC. Symptoms and perceived severity of illness as predictive of treatment for diarrhoea in six Asian and African sites. Soc Sci Med 1996; 43:429-39.

30. Iyun BF, Oke EA. Ecological and cultural barriers to treatment of childhood diarrhea in riverine areas of Ondo State, Nigeria. Soc Sci Med 2000; 50:953-64.

31. Berlinguer G. A doença. São Paulo: Hucitec; 1988.

Artigo recebido: 01/09/2003

Aceito para publicação: 13/02/2004 